
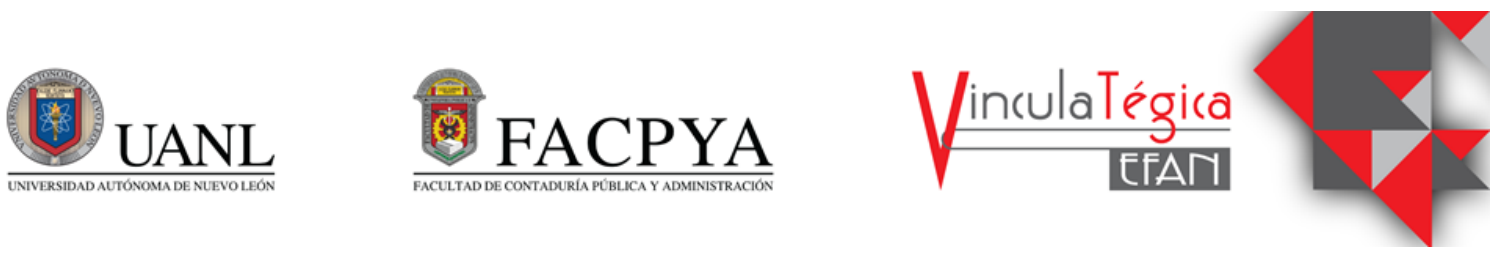

\title{
Diferencia salarial por género en la industria de la construcción en el Área Metropolitana de Monterrey
}

\author{
Martha Ramírez Garza ${ }^{1}$ \\ ${ }^{I}$ Estudiante de Posgrado de la Facultad de Arquitectura en la Universidad Autónoma de Nuevo León, \\ martha.ramirezgrz@uanl.edu.mx, Pedro de Alba S/N, Niños Héroes, Ciudad Universitaria, San Nicolás de \\ los Garza, N.L.
}

Información del artículo revisado por pares

Fecha de aceptación: junio-2021

Fecha de publicación en línea: diciembre-2021

DOI: https://doi.org/10.29105/vtga7.1-102

\section{RESUMEN}

En este estudio se analizó la existencia de una brecha salarial entre hombres y mujeres según su puesto. Se utilizó muestreo bola de nieve, no probabilístico, el cual se basa en referencias de sujetos iniciales para generar sujetos adicionales, de ahí su nombre; aplicado a hombres y mujeres mayores de 22 años, que residen en el área metropolitana de Monterrey (AMM). La hipótesis de esta investigación es que existe una diferencia considerable entre los sueldos y salarios de hombres y mujeres en el AMM, brecha por la discriminación que enfrentamos día con día por el hecho de ser mujeres. La edad, estado civil, percepción económica son algunas variables utilizadas para verificar la presencia de dicha brecha salarial entre hombres y mujeres, así como también la percepción del genero acerca del tema. Los resultados indican que no existe diferencia salarial entre hombres y mujeres, y que incluso las mujeres no tienen esta percepción, la cual se verá desarrollada mas adelante. El trabajo se divide en cinco secciones, la primera es la introducción, en seguida el marco teórico, después hablamos del método seguido de los resultados y al final se da nuestro punto de vista en las conclusiones se presenta como pudiera mejorar el artículo.

Palabras clave: diferencia salarial, género, industria de la construcción, AMM, muestreo no probabilístico.

\begin{abstract}
In this study, the existence of a salary gap between men and women was analyzed according to their position. Non-probabilistic snowball sampling was used, which is based on initial subject references to generate additional subjects, hence its name; applied to men and women over 22 years of age, who reside in the metropolitan area of Monterrey (AMM). The hypothesis of this research is that there is a considerable difference between the wages and salaries of men and women in the AMM. And that this gap is due in part to the discrimination that we face every day for the fact of being women. Age, marital status, economic perception are some variables used to verify the presence of said wage gap between men and women, as well as the perception of gender on the subject. The results indicate that there is no salary difference between men and women, and that even women do not have this perception, which will be developed later. The work is divided into five sections, the first is the introduction, then the theoretical framework, then we talk about the method followed by the results and at the end our point of view is given in the conclusions and how the article could improve.
\end{abstract}

Keywords: wage gap, gender, construction industry, AMM, non-probability sampling.

JEL: J31, M54, M12. 


\section{INTRODUCCIÓN}

Los estereotipos de género influyen de gran manera en los negocios ya que tienen más prioridad para los hombres que las mujeres, esto es base de nuestros aspectos culturales y sociales; así lo dice Ortiz, Picazzo y Alvarado (2020a).

En México el derecho al sufragio universal a las mujeres les otorgó calidad de ciudadanas y, fue hasta 1953; encabezado por Adolfo Ruiz Cortines, (Jaiven, 2015). De acuerdo con los últimos datos de personal ocupado en la construcción, en noviembre de 2019 tenía 55,130 y en noviembre de 2020 , 49,692 personas ocupadas, lo cual refleja una reducción del $9.86 \%$ (Secretaría de la Economía y el Trabajo, SET 2021). En el primer trimestre del 2021, esta industria se vio afectada por la pandemia COVID-19, con una disminución de 1.7 millones de personas en la Población Económicamente Activa (PEA), al pasar de 57.6 millones a 55.9 millones. La población ocupada fue de 53.3 millones de personas, reportando una disminución de 2.4 millones (Instituto Nacional de Estadística y Geografía, INEGI 2021).

En este artículo se compara la teoría de la brecha salarial por género. Para ello, se recabo información mediante una encuesta aplicada vía Google Forms. Como se mencionó, la unidad de análisis fueron los trabajadores del área metropolitana de Monterrey, el AMM es la segunda más poblada del país (López, 2021). La finalidad es verificar la cantidad de trabajadores que se desempeñan en el área de la construcción, y cuántos de ellos son mujeres. Además, se verifica que, ante la necesidad de recortar el equipo de trabajo, la industria decidió por finiquitar inicialmente a mujeres o a hombres.

\section{MARCO TEÓRICO}

En 1916 Hermila Galindo secretaria general de Venustiano Carranza, defendía a las mujeres creando un escrito en el cual se hablaban de los derechos de las mujeres. En 1920 ocurrió un suceso político que hizo que declinara la petición para defender a las mujeres.

Salvador Alvarado, quien estaba frente al gobierno en Mérida Yucatán (1915-1918) habla de la independización de las mujeres en el área económico y para ello metió en su gobierno mujeres capacitadas y así mismo hizo cambios en el código civil para que las mujeres pudieran trabajar fuera de casa. De acuerdo con Jaiven (2015), las mujeres podían salirse de su casa a los 21 años, en la década de los veinte y treinta las mujeres hacían organizaciones para hacer valer sus valores, los cuales eran: igualdad salarial, seguro en el campo laboral, dormitorios, comedores, formación de agrupaciones libertarias.

El consejo feminista mexicano (CFM 1919 - 1925) y el frente único pro derechos de la mujer (FUPFM) son las principales organizaciones que defendían los derechos de las mujeres en su trabajo ya que como se mencionó antes las mujeres no podían alzar su voz ni punto de vista porque no le hacían caso.

En Alemania el 14\% de los astrónomos eran mujeres que trabajaban en talleres artesanales en negocios familiares así lo dice Arauz (2015) esto fue algo que después tuvo como consecuencia la revolución científica que debemos resaltar que no tenían mucho conocimiento del tema lograron estar fuertemente en el campo de la ciencia.

En el porfiriato ya había mujeres que exigían los derechos de las mujeres y que los querían hacer valer, algunas mujeres que estuvieron estas agallas de alzar su voz fueron: Juana Belén Gutiérrez de Mendoza y Dolores Jiménez y Muro. Estas mujeres no permitían que siguieran las injusticias de la sociedad sobre las mujeres. En los noventa surge un movimiento feminista el cual hizo que los derechos de las mujeres fueran tomadas en cuenta en la política nacional. Este grupo de feministas se fue dando gracias a que las mujeres no decidían quedarse más calladas y luchaban por sus derechos y sus opiniones.

\subsection{Brecha salarial.}

Los autores principales de esta teoría son: Blinder (1973), Oaxaca (1973), Ashenfelter, O. and R. Oaxaca (1987), Polachek y Goldin (1987), Psacharopoulos y Tzannatos (1992) y de Blau y Kahn (1996), ellos mencionan que la discriminación y la diferencia salarial están relacionados con la economía de género en México y en ellos se distinguen las variables 
de raza o sexo

En teoría, la diferencia en ingresos, se explica desde tres fuentes: 1) de la diferencia visible de dotaciones en capital humano entre individuos de diferenciados por su sexo; 2) hipótesis de la discriminación en donde no son remunerados de igual manera por diferencias en su productividad; y 3) la diferencia de ingresos entre géneros como una consecuencia estadística de la oferta de trabajo, hipótesis de selección muestral (Heckman, 1979).

\subsection{Evidencia de discriminación salarial en México}

Para la población de 18 años y más con educación superior y su distribución por género, y en campos de formación. Tomando en cuenta la población mayor de 18 años con educación superior en el campo de ingeniería, manufactura y construcción, donde se encuentra que en una población de entre 100,000 y más habitantes se encuentran 482,038 hombres y 444,821 mujeres. Dando un total de 926,859 personas en este rango. Donde solo el $8.46 \%$ son mujeres de todo el total de personas ocupadas. (Encuesta Nacional de Ocupación y Empleo. ENOE, 2021). Lo que evidencia las pocas oportunidades a las mujeres reciben en este rango.

Además, de acuerdo con tablas informativas de Monterrey Nuevo León donde indica que tiene una población ocupada de 1'995,739 personas y desocupada de 92,450. Un $4.42 \%$ de la población desocupada en referencia de la ocupada hasta el cuarto trimestre del 2020. Aun y con COVID-19, los rangos no estaban tan descabellados negativamente en cuanto a trabajo (ENOE, 2021).

Por su parte, Ortiz, Picazzo y Alvarado (2020b) señalan que si existen diferencias en las intenciones de hombres y mujeres en los negocios en México y los motivos de emprender en cualquier negocio (incluido en la construcción) el género es fundamental dependiendo el tipo de mercado, así como la escolaridad, razón del querer iniciar el negocio, la profesión, el número de trabajadores y la industria.
Es importante agregar que es más probable que los hombres tengan puestos importantes, ya que Fuentes y Sánchez (2014) señalan en su artículo que la mujer es menos emprendedora que el hombre, según estudios realizados, y existen evidencias de que las mujeres tienen a no ser emprendedoras. Aunque en el ámbito laboral la mujer ha sumado cada día más presencia, todavía existe dicha brecha considerable en empresas que son creadas por diferentes géneros. Esto se ve evidenciado en los datos económicos, donde se ve evidente que se necesita prestar atención al fenómeno económico social que existe en la actividad empresarial del género femenino. (Fuentes, 2014).

\subsection{Discriminación salarial en México}

$\mathrm{Al}$ analizar los ingresos de hombres y mujeres en el ámbito nacional se encontró que el salario promedio mensual de las mujeres representa $74 \%$ del que reciben los hombres. En la región norte es donde las personas percibieron un salario mayor y la región sur es donde percibieron los menores salarios, recibiendo las mujeres en el norte $80 \%$ del salario de los hombres y en el sur $72 \%$. Al tomar en cuenta la variable profesión, en el país las personas que cuentan con este nivel de preparación profesional de licenciatura reviven mayor salario ya sea visto en valor mensual o por hora.

Sin embargo, las mujeres siguen en desventaja, las profesionistas obtienen $75 \%$ del salario promedio mensual que perciben los hombres en dicha categoría y en lo que respecta a las personas que no cuentan con estudios profesionales, las mujeres reciben $73 \%$ del salario mensual promedio que ganan los hombres. Al analizar las regiones nos damos cuenta que todas existen en la misma situación. (Mendoza y García, 2009).

En el ámbito de la economía las mujeres han sumado su participación laboral. La evidencia mostrada coincide con lo apuntado por Papadópulos y Radakovich (2006) respecto a que las mujeres no sólo han accedido a los Enero - Junio 2017147 estudios superiores en forma creciente, alcanzando un buen desempeño en su rendimiento académico, sino que en algunas 
áreas han sobrepasado los porcentajes de egreso masculino. Pero, a pesar de esto las mujeres siguen recibiendo salarios por debajo de los salarios masculinos sin tener una justificación de productividad que generan para la empresa.

Actualmente, los salarios y ocupaciones "destinadas" para hombres y mujeres nos orillan a recibir ingresos que son visiblemente percibidos en diferencias monetarias. Los estudios nos demuestran que existe una relación directa entre el nivel de escolaridad y los ingresos de los trabajadores, por lo que éstos aumentan en función del número de años de estudio y a los años de experiencia que el trabajo les ha generado. Sin embargo un nivel superior de escolaridad no nos es garantía que los ingresos promedios de hombres y mujeres sean iguales ni que tienda a reducirse la brecha entre los mismos. (Rodríguez, Reyna y Limas, 2017).

\section{MÉTODO}

Se desarrolló una encuesta, se utilizó muestreo bola de nieve, no probabilístico, el cual se basa en referencias de sujetos iniciales para generar sujetos adicionales, de ahí su nombre; el cual se aplicó a personas mayores de 22 años, durante el primer trimestre del año 2021. Los entrevistados se eligieron por su disposición a responder, motivo por el cual no se distribuyeron de manera uniforme en los diferentes municipios de la zona metropolitana de Monterrey. Recaudando un total de 110 encuestas recolectadas de manera digital mediante la aplicación Google Forms por el método antes mencionado, y terminó en esa cantidad de encuestados. El cuestionario está compuesto por 19 preguntas organizadas en 5 secciones.

En el primer bloque se describe el título de la encuesta, así como la finalidad de los datos para temas académicos y poder fomentar a las personas que nos apoyaran con dicha encuesta.

El segundo bloque era de información general, donde recabamos información como edad, sexo, estado civil, entre otras características generales. En el tercero se orientaba más hacia la información laboral, el cuarto bloque se recabo información acerca del puesto que desempeña actualmente en donde labora, así como percepciones mensuales, dato importante para la investigación. Y en el quinto y último bloque hablaba sobre la percepción del ambiente laboral así como del como sentían que se tomaban en cuenta a las mujeres en lo laboral, si creían que existía algún tipo de discriminación o violencia hacia ellas.

A partir de dicha recaudación de fondos, se utilizó la herramienta Stadistical Package of the Social Sciences (SPSS) para la realización de análisis estadísticos descriptivos y de frecuencias. Con la final de verificar como estamos conforme a la hipótesis planteada en un inicio donde estamos de acuerdo que existe una brecha salarial entre el hombre y la mujer.

\section{RESULTADOS}

Del instrumento se obtuvo una muestra de 110 personas encuestadas, variables que se encuentran descritas en la siguiente Tabla 1. Las variables nos sirven para identificar la situación en la que se encuentran las personas entrevistadas y el estatus de sus respuestas que analizaremos en las tablas que continúan más adelante. Es importante recordar que las preguntas no estaban forzadas a responderse, por lo cual habrá algunas variables que no contestaron los encuestados.

Además de analizar desde el punto de vista del área metropolitana de Monterrey, podemos suponer que estamos analizando una gran parte de Nuevo León, ya que la mayor parte de la población está centrada en esta parte, por su industria, por trabajo, y la gran cantidad en la que ha crecido.

La descripción de variables no es más que poner en un contexto de la manera en que se están analizando las variables y que podamos estar tanto el exponente como quien recibe, están teniendo la misma idea y pensamiento al analizar las variables que presentaremos a continuación en la Tabla 1. 
Tabla 1. Descripción de variables

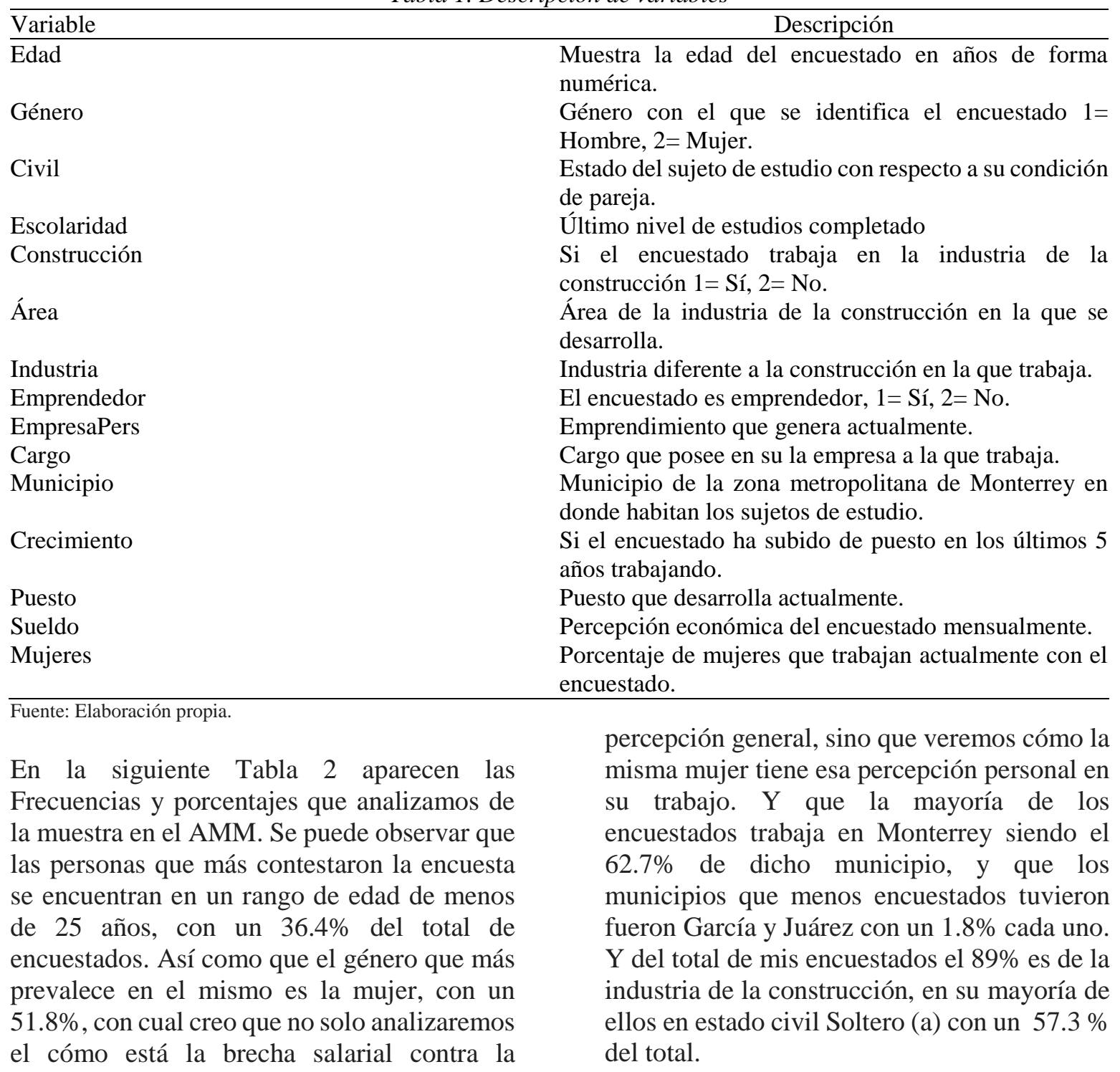


Tabla 2. Variables sociodemográficas de los encuestados y porcentajes

\begin{tabular}{|c|c|c|}
\hline Variable & Frecuencia & Porcentaje \\
\hline \multicolumn{3}{|l|}{$\overline{\text { Edad }}$} \\
\hline Menos de 25 años & 40 & 36.4 \\
\hline De 26 a 35 años & 36 & 32.7 \\
\hline De 36 a 50 años & 24 & 21.8 \\
\hline De 51 o más años & 10 & 9.1 \\
\hline Total & 110 & 100 \\
\hline \multicolumn{3}{|l|}{ Género } \\
\hline Hombre & 53 & 48.2 \\
\hline Mujer & 57 & 51.8 \\
\hline Total & 110 & 100 \\
\hline \multicolumn{3}{|l|}{$\overline{\text { Escolaridad }}$} \\
\hline Preparatoria & 5 & 4.5 \\
\hline Universidad & 83 & 75.5 \\
\hline Diplomados & 4 & 3.6 \\
\hline Maestrías & 18 & 16.4 \\
\hline Total & 110 & 100 \\
\hline \multicolumn{3}{|l|}{ Municipio } \\
\hline Apodaca & 3 & 2.7 \\
\hline Juárez & 2 & 1.8 \\
\hline García & 2 & 1.8 \\
\hline General Escobedo & 3 & 2.7 \\
\hline Guadalupe & 6 & 5.5 \\
\hline Monterrey & 69 & 62.7 \\
\hline Santa Catarina & 3 & 2.7 \\
\hline San Nicolás de los Garza & 8 & 7.3 \\
\hline San Pedro Garza García & 14 & 12.8 \\
\hline Total & 110 & 100 \\
\hline \multicolumn{3}{|l|}{$\overline{\text { Civil }}$} \\
\hline$\overline{\text { Soltero (a) }}$ & 63 & 57.3 \\
\hline Casado (a) & 39 & 35.5 \\
\hline Unión libre & 7 & 6.4 \\
\hline Viudo (a) & 1 & 0.8 \\
\hline Total & 110 & 100 \\
\hline \multicolumn{3}{|l|}{ Construcción } \\
\hline$\overline{\text { Sí }}$ & 89 & 80.9 \\
\hline No & 21 & 19.1 \\
\hline Total & 110 & 100 \\
\hline
\end{tabular}

Fuente: Elaboración propia. 
Tabla 3. Estadísticos descriptivos de las variables

\begin{tabular}{llrrr} 
& Mínimo & Máximo & Media & $\begin{array}{c}\text { Desv. } \\
\text { Desviación }\end{array}$ \\
\hline Puesto & 1 & 7 & 3.61 & 2.009 \\
Sueldo & 1 & 4 & 2.42 & 1.095 \\
Mujeres & 1 & 4 & 2.06 & 1.007 \\
Crecimiento & 1 & 4 & 1.72 & 1.151 \\
\hline
\end{tabular}

Fuente: Elaboración propia

En la Tabla 3 aparecen los estadísticos descriptivos que analizan a la muestra de encuestados, en la cual podemos de acuerdo con la tabla de descripción de variables donde el puesto que es la media está en 3.61 que es más inclinado a Empleado de la construcción, por lo cual los rangos que estamos analizando deben de variar en conjunto con el rango de empleados. Además de que el sueldo radica en 2.2 lo cual quiere decir que están entre $\$ 10,000$ y $\$ 20,000$ pesos de percepción mensual, el cual coincide con el puesto que tuvo la media. De los 110, el 1.72 dice que ha tenido oportunidad de crecimiento, que es que dos veces han subido su sueldo en los últimos 5 años, en lo personal creo que para estar dentro de los rangos de percepción mensual entre los $\$ 10$ mil y \$20 mil, es muy poco solo haber crecido dos veces, que probablemente si tuvieran más oportunidades de crecimiento no estarían ni en el rango de percepción mensual ni en el rango de empleados. Podemos deducir que en remuneraciones estamos dentro de los rangos medios ya que la mayoría de los que contestaron la encuesta no han tenido oportunidad de crecimiento en los últimos 5 años y además de esto están en puestos estándar en la construcción, no puestos grandes como serían las numeraciones 3 jefe de equipo, 4 supervisor o 5 Gerente o encargado. Creo que parte importante de este análisis es en parte por la falta de crecimiento en tanto para hombres como para mujeres. En el siguiente Gráfico 1 aparecen porcentajes de las respuestas a las encuestas en cuanto a si creen que la mujer tiene un menos precio en el trabajo en donde ellos se desarrollan. Tomando en cuenta que cada respuesta tenía a elegir una opción como el porqué de dicha percepción de ellos.

Gráfico 1. Menosprecio de la mujer (\%)

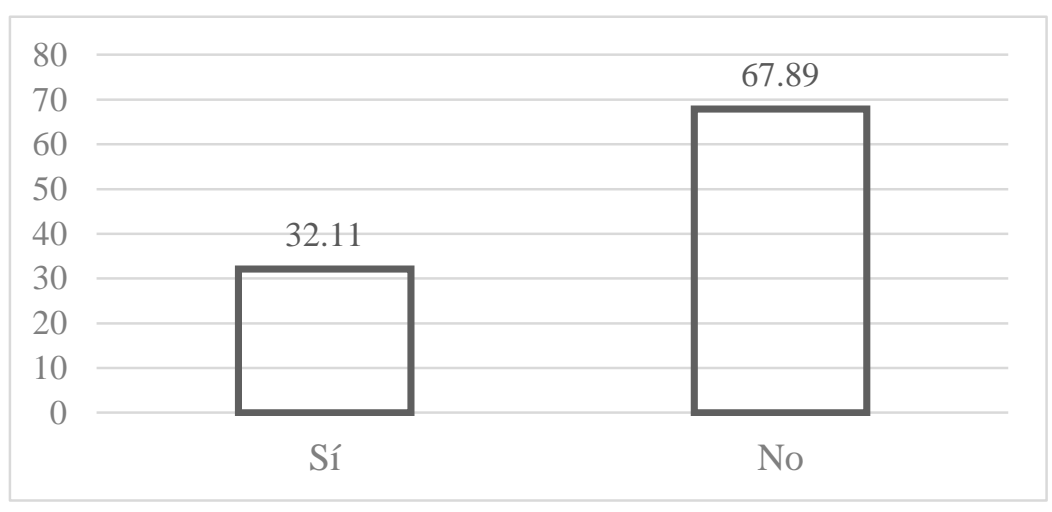

Fuente: Elaboración propia 
En el Gráfico 1, tenemos que de los 110 encuestados, el $67.89 \%$ siente que al género femenino no se hace de menos en sus trabajos, recordando lo que vimos anteriormente, la mayoría de los encuestados fueron mujeres, lo cual quiere decir que ellas también están dentro de este rango.

En el Gráfico 2, tenemos las 4 respuestas que se les dio a elegir si pensaban el rechazo o menosprecio a la mujer, y la respuesta con fue que el $34.28 \%$ de las personas entrevistadas perciben que las mujeres son menospreciadas en sus trabajos ya que tienen menos remuneración económica. Considerando con esto que piensan que no se les ofrecen las oportunidades de crecimiento. Seguido de la discriminación laboral por el simple hecho de ser mujeres, ya que como lo vivimos en la sociedad actual, la mujer es siempre vista como el sexo débil, aun y cuando día con día salen adelante al igual que los hombres. Es impresionante que un número considerable de personas estén de acuerdo que la mujer no gana igual que el hombre.

Gráfico 2. Porque perciben el menosprecio a la mujer (\%)

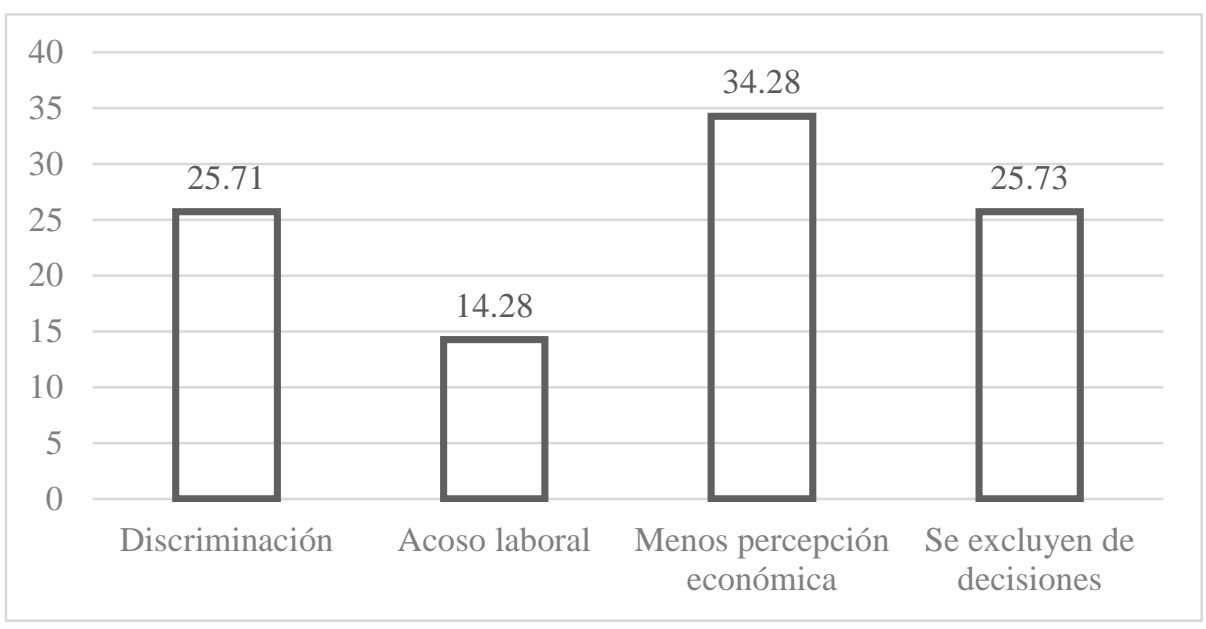

Fuente: Elaboración propia

El Gráfico 3 en donde analizamos las personas que no creen que las mujeres tienen un menosprecio, la respuesta que más porcentaje tuvo, que se les da la misma oportunidad de crecimiento tanto a hombres como a mujeres, con el $38.88 \%$, seguido con el $33.36 \%$ con las personas que piensan que se integran a las mujeres al igual que a los hombres. En lo personal me genera un poco de duda el que nadie seleccionara la respuesta de que tiene $n$ una misma percepción económica, ya que se sabe que las mujeres no ganan igual que los hombres, con el simple hecho de ser mujeres. Recordando que las personas que dijeron que no existe dicha diferencia fue del $67.89 \%$. Lo cual quiere decir que consideran en el siguiente orden de más a menos, que la mujer tiene las mismas oportunidades, que si se integran a las actividades por igual, que se toman en cuanta en las decisiones y por último que reciben la misma percepción económica. 
Gráfico 3. Porque no perciben el menosprecio a la mujer (\%)

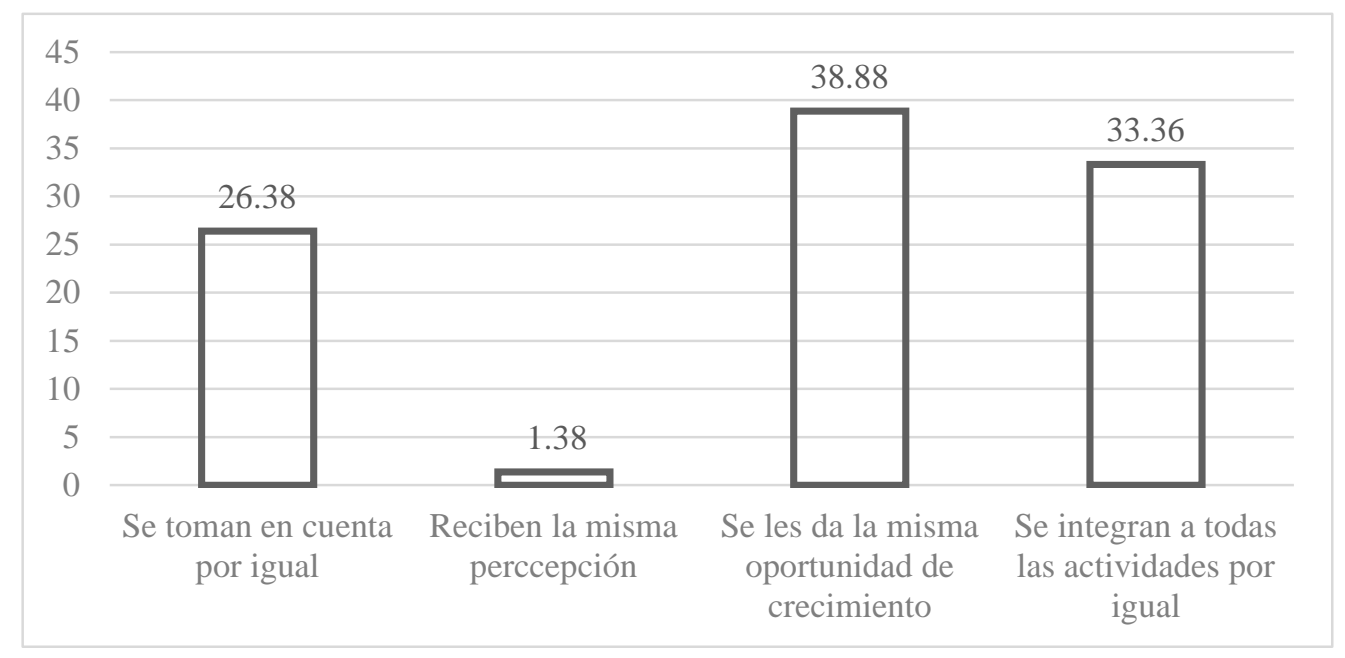

Fuente: Elaboración propia

\section{CONCLUSIONES}

En este artículo se realizó un análisis descriptivo del instrumento aplicado a una muestra de 110 habitantes de la zona metropolitana de Monterrey, donde los encuestados señalaron ser en su mayoría mujeres con un $51.8 \%$ y que trabajan en la industria de la constricción, el 80.9\%.

El $62.7 \%$ de los encuestados tiene su trabajo en el municipio de Monterrey, seguido por el municipio de San Pedro Garza García. La mayoría de estado civil soltero.

El sistema identificó y reportó en su mayoría el sueldo en el rango $\$ 10,000$ y $\$ 20,000$ pesos mensuales. Recordando que el rango que más tenía peso es el de empleado, entonces estamos en los rangos de percepción para nivel empleado.

A lo cual podemos deducir que no existe dicha brecha económica entre hombres y mujeres ya que en primera estancia los datos que analizamos estaban muy parejos en género, y aun con esto las diferencias no marcaron brecha salarial de magnitud.
Y cuando entramos a la percepción de las últimas preguntas, el $67.88 \%$ indica que no percibe dicho menosprecio del género femenino. $\mathrm{Y}$ el $32.11 \%$ dice que si existe dicho menosprecio. Considerando que en su mayoría son mujeres, entonces ellas mismas se dan cuenta que no existe dicha brecha.

De las personas que dijeron que si perciben el menos precio a la mujer en su mayoría siente que es porque existe menos percepción económica. Y los que dicen que no lo perciben señalan que se les da la misma oportunidad de crecimiento en el ámbito laboral. Y solo el $1.38 \%$ percibe que no hay menos precio por que tienen la misma percepción económica.

Es importante señalar que creemos que el instrumento puede mejorar si no solo tomáramos la industria de la construcción, si se analizara solamente en un cierto rango de edades y de puestos para poder comparar con más exactitud con el INEGI o la ENOE. Y además de hacer un muestreo probabilístico para tener exactitud en el tamaño de muestra. 


\section{REFERENCIAS}

Arauz, D. (2015). Primeras mujeres profesionales en México. Instituto Nacional de Estudios Históricos de las Revoluciones de México. En: https://inehrm.gob.mx/work/models/inehrm/Resource/1484/1/images/HistMujeresMexico.pdf

Arceo, E. O. y Campos, R. M. (2014). Evolución de la brecha salarial de género en México. El trimestre económico Vol. LXXXI (3), pp. 619-653 En: https://www.redalyc.org/pdf/313/31340981004.pdf

Fuentes, F. J. y Sánchez, S. M. (2010). Análisis del perfil emprendedor: una perspectiva de género. Estudios de economía aplicada. Vol. 28 (3), pp. 01-27 En: https://www.redalyc.org/pdf/301/30120334014.pdf

Heckman, J. (1979). "Sample Bias As a Specification Error." Econometrica, 47 (1), pp. 153-161.

Instituto Nacional de Estadística y Geografía (2021). Resultados de la encuesta nacional de ocupación y empleo. Nueva edición (ENOEN). Cifras durante el cuarto trimestre de 2020 (115/21). INEGI INFORMA. En: https://www.inegi.org.mx/contenidos/saladeprensa/boletines/2021/enoe_ie/enoe_ie2021_02.p df

Instituto Nacional de Estadística y Geografía (2021). Censo de Población y vivienda 2020. INEGI. En: https://www.inegi.org.mx/programas/ccpv/2020/default.html\#Tabulados

Instituto Nacional de Estadística y Geografía (2021). Encuesta Nacional de Ocupación y Empleo (ENOE), población de 15 años y más de edad. ENOE. En: https://www.inegi.org.mx/programas/enoe/15ymas/\#Datos abiertos

Jaiven, A.L. (2015). La historia de las mujeres. Una nueva corriente historiográfica. Instituto Nacional de Estudios Históricos de las Revoluciones de México. En: https://inehrm.gob.mx/work/models/inehrm/Resource/1484/1/images/HistMujeresMexico.pdf

Lexartza, L, Chaves, M. J., Carcedo, A. y Sánchez, A. (2019). La brecha salarial entre hombres y mujeres en América Latina: En el camino hacia la igualdad Salarial. Lima: OIT.

López, C. (28 de abril de 2021). AMM la segunda más poblada del país según el INEGI. El porvenir. https://elporvenir.mx/local/amm-la-segunda-mas-poblada-del-pais-segun-el-inegi/195272

Martínez, I. \& Acevedo, G. (2004). La brecha salarial en México con enfoque de Género: Capital humano, discriminación y selección muestral. Ciencia UANL. VII (1), pp. 01-02. En: http://eprints.uanl.mx/1521/1/brecha salarial.pdf

Mendoza, J. E. y García, K. J. (2009). Discriminación salarial por género en México. Problemas de desarrollo. Revista Latinoamericana de Economía. 40 (156), pp. 77-99 En: https://www.redalyc.org/pdf/118/11820096006.pdf

Ortiz, J., Picazzo, E. y E. Alvarado. (2020a). Does gender affect business continuity? An analysis with hierarchical modeling. Nova Scientia, 12, (25). pp. 1-17.

Ortiz, J., Picazzo, E. y E. Alvarado. (2020b). Diferencias entre hombres y mujeres en los determinantes de las intenciones de continuidad de los micronegocios en México. Acta Universitaria, Volumen 30. pp. 1-15.

Rodríguez, R. (2015). Los derechos de las mujeres en México, breve recorrido. Instituto Nacional de Estudios Históricos de las Revoluciones de México. En: https://inehrm.gob.mx/work/models/inehrm/Resource/1484/1/images/HistMujeresMexico.pdf

Rodríguez, R. E. y Limas, M. (2017). Análisis de las diferencias salariales y discriminación por género por áreas profesionales en México. Estudios Sociales 27 (49), pp. 121-150 En: https://www.redalyc.org/pdf/417/41749480005.pdf

Secretaría de Economía y Trabajo (2021). N.L. Construcción Personal Ocupado y Remuneraciones Medias. DATA Nuevo León. Secretaría del Trabajo. En: http://datos.nl.gob.mx/n-lconstruccion-personal-ocupado-y-remuneraciones-medias/. 